\title{
Digital Holographic Superresolution by Rotating the Object Wavefield
}

\author{
Bryan M. Hennelly, Thomas J. Naughton, John B. McDonald \\ Department of Computer Science, National University of Ireland, Maynooth, \\ Co. Kildare, Ireland.
}

Using photosensitive recording materials to record holograms ${ }^{1}$ is costly and inflexible. Digital holography ${ }^{2,3},(\mathrm{DH})$ refers to the science of using discrete electronic devices, such as CCDs to record the hologram. In this case reconstruction is performed numerically by simulating the propagation of the wavefield back to the plane of the object. One major advantage of DH over material holography is the ability to used discrete signal processing (DSP) techniques to the recorded signals. In recent years DH has been demonstrated to be a useful method in many areas of optics such as microscopy, deformation analysis, object contouring, particles sizing and position measurement. 'In-line' or 'on-axis' DH refers to the implementation of the original Gabor architecture in which the reference wavefield travels in the same direction as the object wavefield. As in the continuous case this method suffers from poor reconstructed image quality, due to the presence of the intensity terms and the conjugate image that contaminates the reconstructed object image. However it is possible to use DSP techniques to minimise this contamination.

While it is possible to remove the intensity terms with efficient numerical techniques, it remains difficult to remove the conjugate image. This may be achieved using an off-axis recording setup equivalent to that used by Leith and Upatnieks ${ }^{4,5}$. However, this increases the spatial resolution requirements, and limits the system significantly which is undesirable when one considers the already limited resolution of pixilated cameras. An alternative approach known as phase-shifting interferometry has been introduced allowing for an in-line set-up to be used with at least two successive captures and enabling separation of the object wavefield from all of the other terms.

This paper deals with the subject of superresolution in Digital Holography (DH), i.e. increasing the resolution of DH system beyond its limit. The limiting factor regarding resolution in a DH system is the pixel size, which is equal to the smallest resolvable unit. By careful superposition of different digital holograms captured of the same 3-D object, we attempt to increase the resolution of the reconstructed image and equivalently to increase the range of angles of reconstruction. This is accomplished by rotating the input object wavefield either by rotation of the object (it is 2-D) or by rotation of a mirror that is placed between the object and the CCD. Rotating the input wavefield shifts the wavefield in the hologram plane in space and spatial frequency. Therefore, those parts of the hologram field that contained energy at too great an angle for recording and were therefore arranged to be adjacent to and not on the CCD will be shifted in space onto the CCD face and will also be shifted to an angle that can be recorded. We outline a sub-pixel correlation technique to stitch the consecutive holograms together in both the space and spatial frequency domains. Multiple captures enable us to record a DH of large resolution and angle of reconstruction. Storage and reconstruction of the stitched hologram is also discussed and experimental results are given. We use the Wigner Distribution Function to qualify and quantify the method and we explain the improvements accomplished by the system by comparing the numerical aperture before and after the process of superresolution.

Recently Lohmann et. al. offered a novel interpretation of the holographic principle ${ }^{6}$ in terms of the Wigner Distribution Function ${ }^{7}$ (WDF) . This interpretation offers considerable insight into holography in general including DH. In particular, the storage capacity of materials and electronic devices may be examined in relation to the holograms that are to be stored. The WDF is the ideal tool with which to analyze our superresolution process because it allows us to view our signal as a function of space and spatial frequency (angle) simultaneously. The WDF was initially proposed by E. Wigner in 1932 to describe quantum mechanical systems ${ }^{7}$. The WDF is a bilinear representation of a 1D signal. The 2-D 'Wigner Chart' is a simple tool for describing complex optical phenomena in an elegant diagrammatical fashion ${ }^{37}$. We use Wigner analysis to explicitly describe our method. 


\section{DTuD5.pdf}

The greatest obstacle in digital holography is the resolution of the cameras, which is significantly lower than that of the photosensitive materials. The resolution of optical imaging systems in general may be improved upon using superresolution techniques ${ }^{8-11}$ - i.e. by increasing the synthetic aperture of the system in question. Methods have been proposed in the literature to increase the synthetic aperture of digital holographic systems by creating large digital holograms from many different camera positions ${ }^{9-}$ 11. However, these methods are difficult to implement and take considerable time. In a form of incoherent superposition was proposed ${ }^{12,13}$ to increase the synthetic aperture of holographic systems in which the off axis illumination was used to shift signal energy from high spatial frequencies to lower one enabling recording. The idea of incoherent superposition of digital holograms for superresolution has also been proposed ${ }^{14}$ involving the tilting of the illuminated spherical beams that are incident on objects in DH magnification systems allowing for improvements in resolution in the order of 2-3. The method of superresolution outlined in this paper is based on the coherent superposition of digital holograms and can increase the numerical aperture of many digital holography systems by a large factor, the limit of which has yet to be determined

In this paper, we propose the coherent superposition of different holograms of the same object where each hologram corresponds to a different spatial area in the hologram plane. Recording different sections of the hologram plane and using them to create a large mosaic is a form of digital holographic superresolution. The goal is the recording of a large digital hologram, which may enables us to increase the resolution of the reconstructed object wavefield, and therefore the angle of view. All analysis is carried out using the WDF allowing us to simultaneously consider position and spatial frequency. To capture the various holograms we propose one method in particular but we note that one may propose two methods that theoretically achieve the same results and are equivalent to rotation of the CCD. The first method is based on a rotation of our object, assuming the object is 2-D, around the axes of interest effectively shifting the input object wavefield in the spatial frequency domain. The wavefield that is incident on the CCD is therefore shifted in space and in spatial frequency and so the overall bandwidth of each hologram lies within that of CCD and the standard Nyquist sampling theorem can be employed. The second method and the one that we focus primarily on in this paper is based on a rotation of a mirror (or any suitable reflective or refractive device) that is placed between the object and the CCD in the system, see Fig. 1. This method may be applied to 3-D object and brings about the same shift in space and in spatial frequency of the hologram plane as in the 2-D case mentioned above.

In all of these methods the individual holograms that are captured must be aligned relative to one another using position identification algorithms. It is possible that one may determine these shifts with an accurate knowledge of movement of the apparatus in the experimental procedure but this would require a most difficult accuracy. We propose a double sub-pixel correlation technique using the Discrete Fourier Transform (DFT) to be applied in (i) the space domain (using the absolute value of the recorded holograms) and (ii) the spatial frequency domain (using the DFTs of the spatially overlapping portions of the two complex holograms). Once the relative positions of all the holograms (in (i) space and (ii) spatial frequency or angle) have been identified they must be stitched together in some manner. It becomes clear that actually stitching them together in the most obvious manner to create a large planar hologram is not at all efficient when one considers the computer storage of this 'giant' hologram and the numerical reconstruction process that must be applied to it.

\section{REFERENCES}

1. D. Gabor, “A new microscope principle,” Nature 161, pp. 777-778, (1948).

2. L. P. Yaroslavskii and N. S. Merzlyakov, Methods of Digital Holography (Consultants Bureau, 1980).

3. T. M. Kreis, M. Adams, and W. P. O. Juptner, "Methods of digital holography: a comparison," in Optical Inspection and Micromeasurements II, C. Gorecki, ed., Proc. SPIE 3098, 224-233 (1997).

4. E. N. Leith and J. Upatnieks, "Reconstructed wavefronts and communication theory," J. Opt. Soc. Am. 52, pp 1123 (1962).

5. E. N. Leith and J. Upatnieks, "Wavefront reconstruction with continuous-tone objects," J. Opt. Soc. Am. 53, 1377, (1963).

6. Adolf W. Lohmann, Markus E. Testorf, Jorge Ojeda-Castaneda, "Holography and the Wigner function," Proc. SPIE 4737, p. 77-88, Holography: A Tribute to Yuri Denisyuk and Emmett Leith; H. John Caulfield; Ed. 


\section{DTuD5.pdf}

7. E. Wigner, "On the quantum correction for thermodynamic equilibrium," Phys. Rev. 40, pp. 749759, (1932).

8. W. Lukosz, "Optical systems with resolving powers exceedingthe classical limits. II, ” J. Opt. Soc. Am. 57, 932-941 (1967).

9. F. Le Clerc, M. Gross, and L. Collot, "Synthetic aperture experiment in the visible with on-axis digital heterodyne holography," Opt. Lett. 26, 1550-1552 (2001).

10. J. H. Massig, "Digital off-axis holography with a synthetic aperture," Opt. Lett. 27, 2179-2181 (2002).

11. Thomas Kreis, Daniel Kayser, "Resolution increase by aperture synthesis in digital holography," Proc. SPIE 5880, 58800F, Optical Diagnostics; Leonard M. Hanssen, Patrick V. Farrell; Eds.

12. X. Chen and S. R. J. Brueck, "Imaging interferometric lithography: approaching the resolution limits of optics," Opt. Lett. 24, 124-126 (1999).

13. C. J. Schwarz, Y. Kuznetsova, and S. R. J. Brueck, "Imaging interferometric microscopy," Opt. Lett. 28, 1424-1426 (2003).

14. V. Mico, Z. Zalevsky, P. García-Martínez, and J. García, "Superresolved imaging in digital holography by superposition of tilted wavefronts," Appl. Opt. 45, 822-828 (2006).

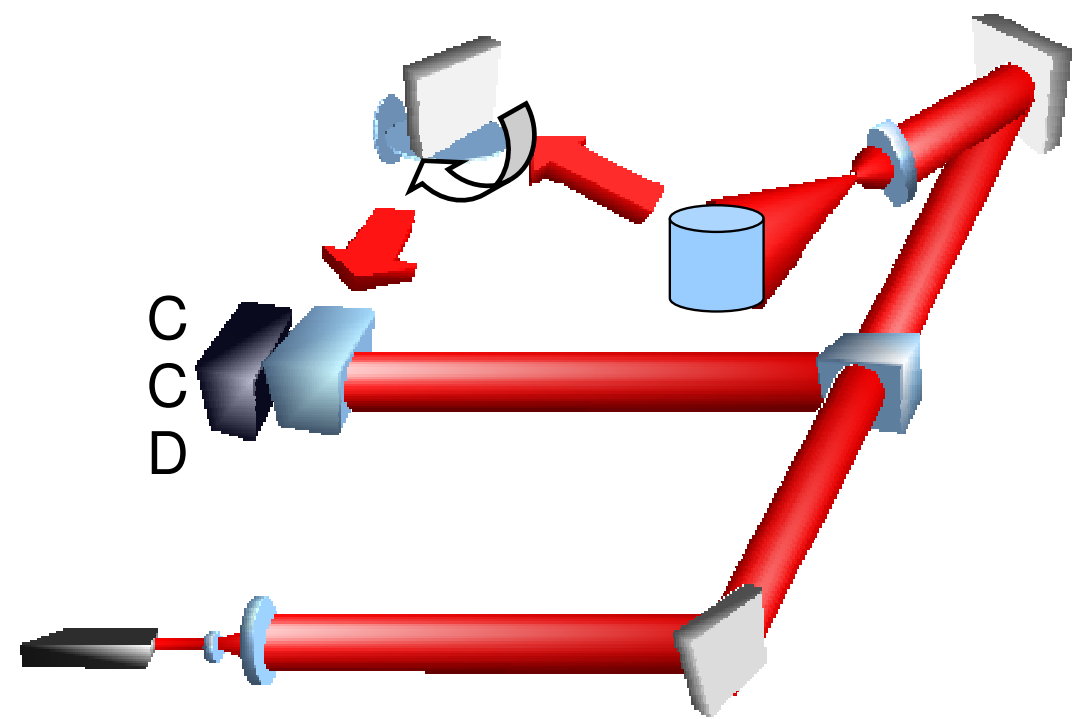

Fig. 1. Architecture for superresolving digital holography. Here our input is a 3-D object. A mirror, positioned close to the object reflects the object wavefield towards the CCD. The mirror can be rotated. 